\title{
ON THE DERIVATION REPRESENTATION OF THE FUNDAMENTAL LIE ALGEBRA OF MIXED ELLIPTIC MOTIVES
}

\author{
S. BAUMARD AND L. SCHNEPS
}

\begin{abstract}
Richard Hain and Makoto Matsumoto constructed a category of universal mixed elliptic motives, and described the fundamental Lie algebra of this category: it is a semi-direct product of the fundamental Lie algebra Lie $\pi_{1}$ (MTM) of the category of mixed Tate motives over $\mathbb{Z}$ with a filtered and graded Lie algebra $\mathfrak{u}$. This Lie algebra, and in particular $\mathfrak{u}$, admits a representation as derivations of the free Lie algebra on two generators. In this paper we study the image $\mathscr{E}$ of this representation of $\mathfrak{u}$, starting from some results by Aaron Pollack, who determined all the relations in a certain filtered quotient of $\mathscr{E}$, and gave several examples of relations in low weights in $\mathscr{E}$ that are connected to period polynomials of cusp forms on $\mathrm{SL}_{2}(\mathbb{Z})$. Pollack's examples lead to a conjecture on the existence of such relations in all depths and all weights, that we state in this article and prove in depth 3 in all weights. The proof follows quite naturally from Ecalle's theory moulds, to which we give a brief introduction. We prove two useful general theorems on moulds in the appendices.
\end{abstract}

\section{INTRODUCTION}

1.1. Motivation. In the unpublished text [12], Hain and Matsumoto define a Tannakian category MEM of mixed elliptic motives, and give a partially conjectural description of its fundamental Lie algebra. The elements of this Lie algebra satisfy certain relations coming from modular forms that seem to be related to other natural appearances of modular forms in the theory of multiple (particularly double) zeta values; see $[14,20]$ for the situation in the dual algebra, or [11] for a cohomological explanation of this phenomenon.

Hain and Matsumoto show that the Lie algebra Lie $\pi_{1}(\mathrm{MEM})$ is a semi-direct product $\mathfrak{u} \rtimes \operatorname{Lie} \pi_{1}(\mathrm{MTM})$, where the right-hand factor is the Lie algebra of the pro-unipotent radical of the fundamental group of the category of mixed Tate motives over $\mathbb{Z}$ and $\mathfrak{u}$ is a weight-graded Lie algebra equipped with a depth filtration, related to $\mathrm{SL}_{2}(\mathbb{Z})$.

Hain and Matsumoto construct a representation Lie $\pi_{1}(\mathrm{MEM}) \rightarrow \operatorname{Der} \operatorname{Lie}[a, b]$. This representation is known to be injective on the subalgebra Lie $\pi_{1}(\mathrm{MTM})^{1}$ and conjectured to be injective on $\mathfrak{u}$. The image of $\mathfrak{u}$, denoted $\mathscr{E}$, is equipped with a natural weightgrading and depth filtration, but is far from free. It was studied by Aaron Pollack [17], who defined a filtration $\Theta$ on $E$ different from the depth filtration, and classified all

\footnotetext{
Date: July 13, 2021.

${ }^{1}$ This result follows from combining standard results from the literature. Indeed, Goncharov constructed a Hopf algebra $\mathcal{M Z}$ of motivic multiple zeta values, and it follows from work of $\mathrm{F}$. Brown ([2]) that Lie $\pi_{1}(\mathrm{MTM})$ is isomorphic to the dual of the quotient of $\mathcal{M Z}$ modulo products. Goncharov also showed that elements of $\mathcal{M Z}$ satisfy the associator relations, which by duality gives an injective map Lie $\pi_{1}(\mathrm{MTM}) \hookrightarrow \mathfrak{g r t}$ into the Grothendieck-Teichmüller Lie algebra. Finally, in [7], Enriquez constructed an explicit injective map from $\mathfrak{g r t}$ into Der Lie $[a, b]$. The injectivity of the map Lie $\pi_{1}(M T M) \hookrightarrow$ Der Lie $[a, b]$ also appears as theorem 3.1 in the recent preprint [4], where it has a very different proof based on substantial work by Hain and Levin-Racinet.
} 
relations in the quotient $\mathscr{E} / \Theta^{3} \mathscr{E}$, showing that in each weight $n$ and depth $d>1$, these relations are in bijection with the period polynomials of the same parity as $d$ associated to modular forms on $\mathrm{SL}_{2}(\mathbb{Z})$.

This leads to two natural questions. Firstly, one may ask whether Pollack's relations in $\mathscr{E} / \Theta^{3} \mathscr{E}$ lift to actual relations in the Lie algebra $\mathscr{E}$. Pollack computed several examples of such relations in low weights, and his observations on these examples lead to a natural hypothesis that we state as Conjecture 1.2 at the end of this section. The conjecture is trivial in depth 2 since a depth 2 relation in $\mathscr{E} / \Theta^{3} \mathscr{E}$ also holds in $\mathscr{E}$. The goal of this paper is to prove the conjecture in depth $3^{2}$.

The second natural question is, in the absence of knowledge about the injectivity of the map $\mathfrak{u} \rightarrow \mathscr{E}$, whether Pollack's relations in $\mathscr{E}$ lift to relations in $\mathfrak{u}$, i.e. whether they are "motivic". Hain recently proved that Pollack's depth 2 relations are motivic, but it remains an open question for $d \geqslant 3$.

The methods we use to prove Conjecture 1.2 are based on the passage from non-commutative to commutative variables via techniques from Ecalle's theory of moulds, to which we give a very brief introduction in section 3. The remarkable advantage of using moulds is that the proof involves not just polynomials such as elements of $\operatorname{Lie}[a, b]$, but rational functions whose denominators play a very useful role.

Acknowledgments. The first author thanks Clément Dupont for useful discussions. We also thank Richard Hain for pointing out an ambiguity in an early version of this paper, as well as the anonymous referee for pointing out that the original proof was more complicated than necessary.

1.2. The Lie algebra $\mathscr{E}:$ definition and statement of results. The free Lie algebra Lie $[a, b]$ is graded by the weight, i.e. the degree of polynomials in $a$ and $b$, and by the depth, i.e. the minimum number of $b$ 's in any monomial.

The weight-grading induces a grading on the Lie algebra Der Lie $[a, b]$ of derivations of Lie $[a, b]$ : a derivation is of weight $n$ if the image of $a$ and $b$ are of weight $n+1$. The depth-grading induces a grading on Der Lie $[a, b]$ : a derivation $D$ is of depth $r$ if $D(a)$ is of homogeneous depth $r$ and $D(b)$ of homogeneous depth $r+1$.

For any word $w=w_{1} \cdots w_{r}$ with $w_{i} \in\{a, b\}$, and any $g \in \operatorname{Lie}[a, b]$, we write $w \cdot g=$ $\operatorname{ad}\left(w_{1}\right) \cdots \operatorname{ad}\left(w_{r}\right)(g)$. Hain and Matsumoto showed that the image $\mathscr{E}$ of $\mathfrak{u}$ is generated by the derivations $\varepsilon_{2 i}$ defined for $i \geqslant 0$ by

$$
\varepsilon_{2 i}(a)=a^{2 i} \cdot b \quad \text { and } \quad \varepsilon_{2 i}(b)=\sum_{j=0}^{i-1}(-1)^{j}\left[a^{j} \cdot b, a^{2 i-1-j} \cdot b\right] .
$$

The $\varepsilon_{2 i}$ all satisfy the relation $\varepsilon_{2 i}([a, b])=0$. The derivation $\varepsilon_{2}$ commutes with all the others, so it plays no role in our investigation of relations in $\mathscr{E}$.

The relations between the brackets of derivations that are the subject of Pollack's work [17] are more intricate than it might seem from their simple definition. In this article, we will concentrate on depth 3 relations, namely relations between derivations of the form $\left[\varepsilon_{2 i},\left[\varepsilon_{2 j}, \varepsilon_{2 k}\right]\right]$ in $\mathscr{E}$.

Let $\mathfrak{s l}_{2} \subset$ Der Lie $[a, b]$ be the Lie algebra generated by $\varepsilon_{0}$ and a second derivation $\varphi_{0}$ defined by $\varphi_{0}(a)=0, \varphi_{0}(b)=a$. Elements of $\mathscr{E}$ that commute with $\varphi_{0}$ are called elements of highest weight of $\mathscr{E}$. The algebra $\mathscr{E}$ is also equipped with a filtration denoted

\footnotetext{
${ }^{2}$ The same statement appears without proof in the online lecture notes [5] by F. Brown.
} 
$\Theta$ (see $[17$, p. 5-7]), which is the filtration induced by the descending central series filtration on the subalgebra of Lie $[a, b]$ generated by the $w \cdot[a, b]$ with $w=a^{i} b^{j} \in \mathbb{Q}\langle a, b\rangle$.

We can now give a precise formulation of Pollack's main theorem. Let $\mathscr{E}_{2} \subset \mathscr{E}$ be the subspace spanned by elements of the form

$$
\left[\varepsilon_{0}^{i} \cdot \varepsilon_{p}, \varepsilon_{0}^{j} \cdot \varepsilon_{q}\right], \quad p, q>2 \text { even. }
$$

Then for all depths $d>1$, Pollack shows that the depth $d$ elements

$$
h_{p, q}^{d}=\sum_{i+j=d-2}(-1)^{i} \frac{(d-2) !}{\left(\begin{array}{c}
p \\
i
\end{array}\right)\left(\begin{array}{c}
q \\
j
\end{array}\right)}\left[\varepsilon_{0}^{i} \cdot \varepsilon_{p+2}, \varepsilon_{0}^{j} \cdot \varepsilon_{q+2}\right] .
$$

span the highest weight part of the subspace $\mathscr{E}_{2}$. Pollack's main theorem identifies the relations between these elements in the quotient $\mathscr{E} / \Theta^{3} \mathscr{E}$ :

Theorem 1.1 ([17, Thm. 2]). Let $D$ be a highest weight element of $\mathscr{E}_{2}$, of weight $n$ and depth $d$. Then $D \equiv 0\left[\Theta^{3} \mathscr{E}\right]$ if and only if it is of the form

$$
\mathrm{R}_{f, d}=\sum_{p+q=n-4} r_{p-d+2}(f) h_{p, q}^{d},
$$

where $r_{i}(f)$ is the $i$-th period (in the sense of $[15, \S 11]$ ) of a modular form $f$ of weight $n-$ $2 d+2$ on $\mathrm{SL}_{2}(\mathbb{Z})$.

If $f$ is a modular form of modular weight $k$, then the element $\mathrm{R}_{f, d} \in \mathscr{E}_{2}$ is of depth $d$ and weight $n=k+2 d-1$. The depth 2 and 3 elements corresponding to the weight 12 cusp form known as the Ramanujan $\Delta$ are given by

$$
\begin{gathered}
\mathrm{R}_{\Delta, 2}=h_{2,8}^{2}-3 h_{4,6}^{2} \equiv 0\left[\Theta^{3} \mathscr{E}\right] \\
\text { and } \quad \mathrm{R}_{\Delta, 3}=4 h_{2,10}^{3}-25 h_{4,8}^{3}+21 h_{6,6}^{3} \equiv 0\left[\Theta^{3} \mathscr{E}\right] .
\end{gathered}
$$

Theorem 1.1 shows that, as also occurs in other situations (cf. [13, 20, 16, 12, $3]$ ) related to the theory of multiple zeta values, periods of modular forms appear as coefficients of relations in $\mathscr{E} / \Theta^{3} \mathscr{E}$. Pollack asked the following question: when do these relations in $\mathscr{E} / \Theta^{3}$ lift to actual relations in $\mathscr{E}$ ? Explicit calculation shows that this is not the case in general for the relations associated to the Eisenstein series for even $d$ : for example, the relation $h_{d, n+d-2}^{d+2} \equiv 0\left[\Theta^{3} \mathscr{E}\right]$ associated to the series $E_{n+4}$ does not lift to an identity in $\mathscr{E}$. However, Pollack observed on several examples that the relations coming from cusp forms do seem to lift to relations in $\mathscr{E}$. For example, the relation $\mathrm{R}_{\Delta, 3} \equiv 0\left[\Theta^{3} \mathscr{E}\right]$ admits the lifting

$$
4 h_{2,10}^{3}-25 h_{4,8}^{3}+21 h_{6,6}^{3}=-\frac{345}{8}\left[\varepsilon_{6},\left[\varepsilon_{6}, \varepsilon_{4}\right]\right]+\frac{231}{20}\left[\varepsilon_{4},\left[\varepsilon_{8}, \varepsilon_{2}\right]\right] .
$$

We can thus formulate an explicit conjecture framing an answer to Pollack's question as follows.

Conjecture 1.2. Let $k$ be an integer, and let $f$ be a cusp form of weight $k$ for $\mathrm{SL}_{2}(\mathbb{Z})$. Then there exists a linear combination $T$ of brackets of $\varepsilon_{2 i}$, containing at least three $\varepsilon_{2 i}$ with $i>1$, such that $\mathrm{R}_{f, d}=T$.

The goal of this article is to prove this conjecture in the case $d=3$. The theorem can be stated directly in this situation as follows.

Theorem 1.3. Fix a positive integer $n$, and let $D \in \mathscr{E}$ be a linear combination of terms of the form $\left[\varepsilon_{2 i},\left[\varepsilon_{0}, \varepsilon_{2 j}\right]\right]$ with $i, j \geqslant 2$. Assume that $D \in \Theta^{3} \mathscr{E}$. Then $D$ can be written as a linear combination of terms of the form $\left[\varepsilon_{2 r},\left[\varepsilon_{2 s}, \varepsilon_{2 t}\right]\right]$ with $r, s, t \geqslant 2$. 
We observe in passing that $\varepsilon_{2}$ never appears in any brackets of $\varepsilon_{2 i}$ because, as is easily seen by hand, it is central in $\mathscr{E}$. Our proof is based on three ingredients: first of all, a reformulation in terms of polynomial algebra of the condition that a derivation in $\mathscr{E}$ belongs to $\Theta^{3} \mathscr{E}$ (section 2), secondly, a theorem due to Goncharov characterizing certain types of Lie elements in depth 3 (end of section 2), and thirdly, the passage to Ecalle's language of moulds (section 3) which allows us to make interesting use of rational functions and denominators. Two particularly useful theorems from section 3 , one in mould theory and the other concerning the translation of the Lie algebra $\mathscr{E}$ into mould theory, are proved in appendices, and the proof of the main theorem is given in section 4 .

\section{A reformulation of Pollack's property}

2.1. Properties of Lie polynomials. The following definition gives a key useful property of elements of $\mathscr{E}$.

Definition 2.1. The endomorphism push of the vector space $\mathbb{Q}\langle a, b\rangle$ is defined by its value on monomials, given by

$$
\operatorname{push}\left(a^{i_{0}} b \cdots a^{i_{r-1}} b a^{i_{r}}\right)=a^{i_{r}} b a^{i_{0}} b \cdots b a^{i_{r-1}} .
$$

A polynomial $f$ is said to be push-invariant if $\operatorname{push}(f)=f$.

It is known that push-invariant Lie polynomials are exactly the values at $a$ of derivations that are zero on $[a, b]$; we recall this and another useful characterization in the following proposition.

Proposition 2.2 ([21, Thm. 2.1]). Let $P \in \operatorname{Lie}[a, b]$, and assume that $P$ is of homogeneous degree $\geqslant 2$.

i) The polynomial $P$ is push-invariant if and only if there exists an element $Q$ in Lie $[a, b]$ such that $[P, b]+[a, Q]=0$, in other words the derivation $D$ defined by $D(a)=P, D(b)=Q$ satisfies $D([a, b])=0$. If such a $Q$ exists then it is unique.

ii) There exists a polynomial $Q \in \operatorname{Lie}[a, b]$ such that $P=[a, Q]$ if and only if $P$ does not contain any monomials starting and ending in $b$.

Corollary 2.3. Let $D \in \mathscr{E}$. Then $D(a)$ is a push-invariant polynomial.

Indeed, since $D \in \mathscr{E}$, we have $D([a, b])=[a, D(b)]+[D(a), b]=0$, so $D(a)$ is pushinvariant by Proposition 2.2 (i).

We can now give the reformulation of Pollack's property that will serve the purposes of our proof.

Proposition 2.4. Let $D$ be a linear combination of terms of the form $\left[\varepsilon_{2 i},\left[\varepsilon_{0}, \varepsilon_{2 j}\right]\right]$ with $i, j>1$. If the derivation $D$ lies in $\Theta^{3} \mathscr{E}$, there exists a Lie polynomial $Q \in \operatorname{Lie}[a, b]$ such that $D(a)=[a, Q]$.

Proof. By the definition of the filtration $\Theta$, the derivation $D$ lies in $\Theta^{3} \mathscr{E}$ if and only if $D(a)$ can be written as a linear combination of terms of the form $\left[a^{i} \cdot b,\left[a^{j} \cdot b, a^{k} \cdot b\right]\right]$ with $i, j$ and $k$ strictly positive. Thus we can assume

$D(a)=\left(a^{i} \cdot b\right)\left(a^{j} \cdot b\right)\left(a^{k} \cdot b\right)-\left(a^{i} \cdot b\right)\left(a^{k} \cdot b\right)\left(a^{j} \cdot b\right)-\left(a^{j} \cdot b\right)\left(a^{k} \cdot b\right)\left(a^{i} \cdot b\right)+\left(a^{k} \cdot b\right)\left(a^{j} \cdot b\right)\left(a^{i} \cdot b\right)$.

Then the monomials ending with $b$ arise only from the terms

$$
\left(a^{i} \cdot b\right)\left(a^{j} \cdot b\right) a^{k} b-\left(a^{i} \cdot b\right)\left(a^{k} \cdot b\right) a^{j} b-\left(a^{j} \cdot b\right)\left(a^{k} \cdot b\right) a^{i} b+\left(a^{k} \cdot b\right)\left(a^{j} \cdot b\right) a^{i} b .
$$


In particular this shows that no monomial in $D(a)$ can end in $b^{2}$. Thus since $D(a)$ is a push-invariant polynomial by Corollary 2.3 , no monomial of $D(a)$ can start and end with $b$. Then by Proposition 2.2 (ii), it follows that $D(a)$ is of the form $[a, Q]$.

2.2. A theorem of Goncharov. We end this section by giving a result of Goncharov which will be essential to the proof of our main theorem.

Definition 2.5. Set $b_{i}=a^{i-1} b$ for $i \geqslant 1$. For every polynomial $P \in \operatorname{Lie}[a, b]$, let $P_{*}$ be the polynomial obtained from $P$ by forgetting all the words ending in $a$ and adding on the term $\sum_{i \geqslant 1} \frac{(-1)^{i-1}}{i}\left(P \mid a^{i-1} b\right) b^{i}$, where $(P \mid w)$ denotes the coefficient of the word $w$ in the expanded polynomial $P$, where a Lie polynomial written as a sum of Lie brackets is expanded as an ordinary polynomial in $a, b$ via the rule $[a, b]=a b-b a$, with $\operatorname{Lie}[a, b]$ considered as a subspace of its universal enveloping algebra $\mathbb{Q}\langle a, b\rangle$. The polynomial $P$ is said to satisfy the linearized double shuffle relations if, rewriting $P_{*}$ as a polynomial in the $b_{i}$, it lies in $\operatorname{Lie}\left[b_{1}, b_{2}, \ldots\right]$. Following the notation of [3], we write $\mathfrak{L s}_{\mathfrak{s}}$ for the space of polynomials satisfying the linearized double shuffle relations. The space $\mathfrak{l}_{\mathfrak{s}}$ is weight and depth graded, with weight being the degree of the polynomials and depth the number of $b$ 's in each monomial. We write $\mathfrak{s}_{n}^{d}$ for the part of weight $n$ and depth $d$. These spaces are also studied in [14], which uses the notation $D S h_{d}(n-d)$ for $\mathfrak{l s}_{n}^{d}$.

To every $P \in \operatorname{Lie}[a, b]$ we associate a derivation $D_{P}$ of $\operatorname{Lie}[a, b]$ by setting $D_{P}(a)=0$ and $D_{P}(b)=[b, P]$. The Lie bracket on derivations defines another Lie bracket on the underlying vector space of $\operatorname{Lie}[a, b]$, the Poisson bracket, denoted $\{\cdot, \cdot\}$ and given explicitly by

$$
\{P, Q\}=[P, Q]+D_{P}(Q)-D_{Q}(P),
$$

which is nothing other than an expression for the usual bracket of derivations: namely, the identity $\left[D_{P}, D_{Q}\right]=D_{\{P, Q\}}$ holds for every polynomials $P$ and $Q$.

The following theorem was proven in $[9, \S 7]$ (but see also $[3,7.3]$ for a clearer explanation). The point that will be essential in the proof of our main theorem is that the powers of $\operatorname{ad}(a)$ that appear in the statement are all $\geqslant 2$.

Theorem 2.6. Let $P \in \operatorname{Lie}[a, b]$ be a polynomial of homogeneous depth 3 satisfying the linearized double shuffle relations. Then $P$ is a linear combination of terms of the form

$$
\left\{\operatorname{ad}(a)^{2 r}(b),\left\{\operatorname{ad}(a)^{2 s}(b), \operatorname{ad}(a)^{2 t}(b)\right\}\right\}
$$

with $r, s, t \geqslant 2$.

\section{Moulds}

In this section we collect some of the basic results and definitions of Ecalle's theory of moulds, which is the framework in which we will prove the main results. For a grand overview of Ecalle's theory containing the requisite statements and definitions, see [6], and for a more detailed introduction to the part of mould theory specifically concerning multiple zeta values and complete proofs of the basic results, see [22].

3.1. Definition. A mould is a collection $\left(A_{r}\right)_{r \geqslant 0}$ where each $A_{r}$ is a function of $r$ commutative variables $u_{i}$ over a given field, which here we will take to be $\mathbb{Q}$. In particular, when $r=0, A_{0}(\emptyset)$ is a constant in $\mathbb{Q}$. The notation being redundant, we will often write $A\left(u_{1}, \ldots, u_{r}\right)$ rather than $A_{r}\left(u_{1}, \ldots, u_{r}\right)$. We will also consider moulds in variables $v_{i}$.

Ecalle defines the following operations and properties: 
- the ari-bracket, a Lie bracket on the space of moulds in the $u_{i}$ (resp. in the $v_{i}$ ) satisfying $A(\varnothing)=0$. Under this bracket, said spaces become Lie algebras denoted ARI (resp. $\overline{\mathrm{ARI}})^{3}$. We write $\mathrm{ARI}^{\mathrm{pol}}$ (resp. $\overline{\mathrm{ARI}}^{\mathrm{pol}}$ ) for the spaces of polynomial-valued moulds; it follows directly from the definition of the aribrackets on ARI (resp. ARI) that these subspaces are actually Lie subalgebras. We recall the explicit expression for the ari-bracket in Appendix A (see [6], [18] or $[22])$;

- the swap, an involutive variable-change map from ARI to $\overline{\mathrm{ARI}}$, defined by

$$
\operatorname{swap}(A)\left(v_{1}, \ldots, v_{r}\right)=A\left(v_{r}, v_{r-1}-v_{r}, \ldots, v_{1}-v_{2}\right) .
$$

From this expression it is clear that $A$ is rational or polynomial-valued, then so is $\operatorname{swap}(A)$;

- the push, a cyclic variable-change operator acting on moulds in ARI by

$$
\operatorname{push}(A)\left(u_{1}, \ldots, u_{r}\right)=A\left(-u_{1}-\cdots-u_{r}, u_{1}, \ldots, u_{r-1}\right) .
$$

We write $\mathrm{ARI}_{\text {push }}$ for the space of moulds that are invariant under the push operator;

- the notion of alternality, a mould $A$ in ARI (resp. in $\overline{\mathrm{ARI}}$ ) being said to be alternal if for each $r>1, A$ satisfies

$$
\sum_{\substack{\sigma \in \mathfrak{S}_{r} \\ \sigma(1)<\cdots<\sigma(s) \\ \sigma(s+1)<\cdots<\sigma(r)}} A\left(u_{\sigma^{-1}(1)}, \ldots, u_{\sigma^{-1}(r)}\right)=0,
$$

(resp. the same condition with $u_{i}$ replaced by $v_{i}$ ). Finally, we say that a mould is bialternal if both $A$ and $\operatorname{swap}(A)$ are alternal. We write $\mathrm{ARI}_{\mathrm{al} / \mathrm{al}}$ for the space of bialternal moulds.

As can be seen in several recent articles $[6,14,3]$, the passage to commutative variables can be very useful in studying algebras that are described in terms of noncommutative variables. By Lazard elimination, the Lie algebra Lie $[a, b]$ can be written as a direct sum

$$
\operatorname{Lie}[a, b]=\mathbb{Q} a \oplus \operatorname{Lie}\left[C_{1}, C_{2}, \ldots\right],
$$

where $C_{i}=\operatorname{ad}(a)^{i-1}(b)$ and the right-hand Lie algebra is the free Lie algebra on the $C_{i}$. Let $\mathbb{Q}\left\langle C_{1}, C_{2}, \ldots\right\rangle$ denote the free non-commutative polynomial ring on the $C_{i}$, and for each $r \geqslant 1$, let $\mathbb{Q}_{r}\left\langle C_{1}, C_{2}, \ldots\right\rangle$ denote the subspace of $\mathbb{Q}\left\langle C_{1}, C_{2}, \ldots\right\rangle$ spanned by the depth $r$ monomials $C_{a_{1}} \cdots C_{a_{r}}$. Define a linear map from $\mathbb{Q}_{r}\left\langle C_{1}, C_{2}, \ldots\right\rangle$ to $\mathbb{Q}\left[u_{1}, u_{2}, \ldots\right]$, where the $u_{i}$ are commutative variables, by

$$
\text { ma : } C_{i_{1}} \cdots C_{i_{r}} \mapsto(-1)^{i_{1}+\cdots+i_{r}-r} u_{1}^{i_{1}-1} \cdots u_{r}^{i_{r}-1} .
$$

If $P \in \operatorname{Lie}\left[C_{1}, C_{2}, \ldots\right]$, we write $P=\sum_{r \geqslant 1} P^{r}$ where each $P^{r}$ denotes the part of $P$ that is homogeneous of depth $r$; then ma extends to a map ma : $\operatorname{Lie}\left[C_{1}, C_{2}, \ldots\right] \rightarrow$ ARI by taking ma $(P)$ to be the mould whose depth $r$ part is given by $\operatorname{ma}\left(P^{r}\right)$.

Under the map ma, properties of a Lie polynomial translate into properties of the associated mould. We assemble the most useful ones in the following theorem.

\footnotetext{
${ }^{3}$ Ecalle uses ARI for bimoulds, which are functions of two sets of variables $u_{i}$ and $v_{i}$; in the framework of this article we never use actual bimoulds (i.e. functions that are non-trivial in both families), but there are situations in which they are very useful.
} 


\section{Theorem 3.1.}

i) The map ma transports the Poisson bracket onto the ari-bracket, that is $\mathrm{ma}(\{f, g\})=$ $\operatorname{ari}\left(\mathrm{ma}_{f}, \mathrm{ma}_{g}\right)$, and restricts to a Lie algebra isomorphism from Lie $[a, b]$ to the subalgebra $\mathrm{ARI}_{\mathrm{al}}^{\mathrm{pol}}$ of alternal polynomial-valued moulds in the $u_{i}$.

ii) A polynomial $P \in \operatorname{Lie}[a, b]$ is push-invariant if and only if $\operatorname{ma}(P)$ is a pushinvariant mould, and the space $\mathrm{ARI}_{\text {push }}$ forms a Lie algebra under the ari-bracket.

iii) Let $Q \in \operatorname{Lie}[a, b]$ and $P=[a, Q]$. Then for each $r \geqslant 1$, we have

$$
\operatorname{ma}(P)\left(u_{1}, \ldots, u_{r}\right)=-\left(u_{1}+\cdots+u_{r}\right) \operatorname{ma}(Q)\left(u_{1}, \ldots, u_{r}\right) .
$$

iv) The map ma restricts to an isomorphism

$$
\mathfrak{l} \rightarrow \mathrm{ARI}_{\underline{\mathrm{al}} / \underline{\mathrm{al}}}^{\mathrm{pol}},
$$

of image the set of moulds in $\mathrm{ARI}_{\mathrm{al} / \mathrm{al}}^{\mathrm{pol}}$ whose depth 1 part is an even function.

v) The space $\mathrm{ARI}_{\underline{a l} / \underline{a l}}$ is a Lie algebra under the ari-bracket, and is contained in $\mathrm{ARI}_{\text {push }}$.

All these results constitute a standard part of mould theory, and can be found scattered through various texts (see [6] for the statements not concerning Lie $[a, b]$, or $[18,1]$; see $[22]$ for a basic introduction to the domain containing complete proofs.)

Definition 3.2. For each $r \geqslant 1$, let

$$
\text { Д }_{r}\left(u_{1}, \ldots, u_{r}\right)=u_{1} \cdots u_{r}\left(u_{1}+\cdots+u_{r}\right) .
$$

In particular $Д_{1}\left(u_{1}\right)=u_{1}^{2}$. Let $\mathrm{ARI}^{\text {sing }}$ denote the space of rational-function valued moulds $A$ such that $\beth_{r} A_{r}\left(u_{1}, \ldots, u_{r}\right)$ is polynomial for each $r \geqslant 1$.

The first main result of this article is the following theorem, which is a key result in the application of mould theory to elliptic motives. Its rather lengthy proof is deferred to Appendix A.

Theorem 3.3 ([1, th. 4.45]). The space

$$
\mathrm{ARI}_{\underline{\underline{\mathrm{al}}} / \underline{\mathrm{al}}}^{\text {sing }}=\mathrm{ARI}_{\underline{\underline{a}} / \underline{\mathrm{al}}} \cap \mathrm{ARI}^{\mathrm{sing}}
$$

is a Lie algebra under the ari-bracket.

Remark. It is shown in [22], Corollary 3.3.4, that the ari-bracket restricted to polynomialvalued moulds corresponds to the Poisson (or Ihara) bracket. Therefore it follows from (a graded version of) Racinet's famous theorem (cf. [18]) on the Lie algebra structure of the double shuffle Lie algebra that the linearized double shuffle space is also Lie algebra under the Poisson bracket, or equivalently, in mould language, that the space of polynomial bialternal moulds, $\mathrm{ARI}_{\underline{\mathrm{al}} / \text { al }}^{\mathrm{pol}}$, forms a Lie algebra under the ari-bracket. But Theorem 3.3 above generalizes this statement to the much larger space $\mathrm{ARI}_{\underline{\text { al }} / \text { al }}^{\text {sing }}$, which contains moulds with denominators of type $\beth_{r}$, so the key new point (cf. Appendix) is the proof that the ari-bracket of two such moulds has the same type of denominator.

Definition 3.4. For $i \geqslant-1$, let $U_{2 i}$ be the mould such that $U_{2 i}\left(u_{1}\right)=u_{1}^{2 i}$ in depth 1 , and $U_{2 i}\left(u_{1}, \ldots, u_{r}\right)=0$ for every depth $r \neq 1$ (we say that $U_{2 i}$ is concentrated in depth 1). Let $\mathcal{U}$ be the Lie subalgebra of ARI generated by the $U_{2 i}$. Since $\beth_{1}=u_{1}^{2}$, we have $U_{2 i} \in \mathrm{ARI}^{\text {sing }}$ for $i \geqslant-1$, so thanks to Theorem 3.3, we have the inclusion $\mathcal{U} \subset$

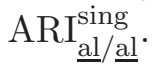


Let $\operatorname{Der}^{0} \operatorname{Lie}[a, b]$ denote the subspace of Der Lie $[a, b]$ consisting of the derivations that kill $[a, b]$, and such that the value of the derivation on $a$ has no linear term in $a$. For every polynomial $F \in \operatorname{Lie}[a, b]$, let $\operatorname{Da}(F)$ denote the mould which is given in depth $r$ by

$$
\operatorname{Da}(F)\left(u_{1}, \ldots, u_{r}\right)=\left(1 / Д_{r}\right) \operatorname{ma}(F)\left(u_{1}, \ldots, u_{r}\right) .
$$

Define a map $\Psi: \operatorname{Der}^{0} \operatorname{Lie}[a, b] \rightarrow$ ARI by

$$
\Psi(D)=\mathrm{Da}(D(a)) .
$$

The following proposition gives the key relationship between brackets of derivations of Lie $[a, b]$ and the ari-bracket on moulds.

Theorem 3.5. The map $\Psi$ is an injective Lie algebra morphism, i.e.

$$
\Psi\left(\left[D_{1}, D_{2}\right]\right) \mapsto \operatorname{ari}\left(\operatorname{Da}\left(D_{1}(a)\right), \operatorname{Da}\left(D_{2}(a)\right)\right) .
$$

The rather long proof of this theorem is deferred to Appendix B. Note however that the injectivity is easy, since $D(a)$ can be recovered from $\mathrm{Da}(D(a))$, and it follows from Proposition 2.2 that a derivation in $\operatorname{Der}^{0} \operatorname{Lie}[a, b]$ is determined by its value on $a$.

The next result ${ }^{4}$ uses this proposition to show that $\mathcal{U}$ is isomorphic to Pollack's Lie algebra $\mathscr{E}$.

Corollary $3.6([8])$. We have $\Psi\left(\varepsilon_{2 i}\right)=U_{2 i-2} \in \mathcal{U}$ for all $i \geqslant 0$, and the map $\Psi$ induces an isomorphism $\mathscr{E} \simeq \mathcal{U}$ of Lie algebras.

Proof. Since $\varepsilon_{2 i}(a)=\operatorname{ad}(a)^{2 i}(b)=C_{2 i+1}$, the mould ma( $\left.\varepsilon_{2 i}\right)$ is the mould in depth 1 that takes the value $u_{1}^{2 i}$, so $\Psi\left(\varepsilon_{2 i}\right)=\mathrm{Da}(D(a))=\mathrm{Da}\left(C_{2 i+1}\right)=U_{2 i-2}$. Then by Theorem 3.5, the map $\Psi$ restricted to the Lie algebra $\mathscr{E} \subset \operatorname{Der}^{0} \operatorname{Lie}[a, b]$ generated by the $\varepsilon_{2 i}$ yields a Lie algebra isomorphism to $\mathcal{U}$.

\section{Proof of the main Result}

We begin by translating Theorem 2.6 to a statement on moulds.

Theorem 4.1. Suppose $A$ is a bialternal polynomial-valued mould concentrated in depth 3. Then $A$ lies in the Lie algebra $\mathcal{U}$, and more precisely it can be written as a linear combination of moulds of the form $\operatorname{ari}\left(U_{2 r}, \operatorname{ari}\left(U_{2 s}, U_{2 t}\right)\right)$ with $r, s, t \geqslant 1$.

Indeed, by (i) of Theorem 3.1, the map ma gives an isomorphism from the space of depth 3 Lie polynomials in $\mathfrak{l}_{\mathfrak{s}}$ to the space of depth 3 moulds in $\mathrm{ARI}_{\mathrm{al} / \mathrm{al}}^{\mathrm{pol}}$, and by (iv) of the same Theorem, since $\operatorname{ma}\left(\operatorname{ad}(a)^{2 r+2}(b)\right)=U_{2 r}$, we have

$$
\operatorname{ma}\left(\left\{\operatorname{ad}(a)^{2 r+2}(b),\left\{\operatorname{ad}(a)^{2 s+2}(b), \operatorname{ad}(a)^{2 t+2}(b)\right\}\right\}\right)=\operatorname{ari}\left(U_{2 r}, \operatorname{ari}\left(U_{2 s}, U_{2 t}\right)\right),
$$

so the statement of Theorem 4.1 is equivalent to that of Theorem 2.6.

We can now prove the main result of this article.

\footnotetext{
${ }^{4}$ This result is also given in $\S 4$ of the preprint [8] by B. Enriquez from January 2013, with a brief indication in lieu of proof. Brown (cf [5], [4]) studies a Lie algebra denoted pls (polar linearized double shuffle) which is isomorphic to swap $\left(\mathrm{ARI}_{\mathrm{al} / \mathrm{al}}^{\mathrm{sing}}\right)$. In the recent preprint [4], Brown mentions without proof the analogous result to Theorem 3.3 for $\mathfrak{p l s}$ (see Definition 4.5), i.e. that $\mathfrak{p l s}$ is closed under the "linearized Ihara bracket". He also gives results that are analogous to Theorem 3.5 and Corollary 3.6.
} 
Theorem 4.2. Fix a positive integer $n$, and let $D \in \mathscr{E}$ be a linear combination of terms of the form $\left[\varepsilon_{2 i},\left[\varepsilon_{0}, \varepsilon_{2 j}\right]\right]$ with $i, j \geqslant 2$. Assume that $D \in \Theta^{3} \mathscr{E}$. Then $D$ can be written as a linear combination of terms of the form $\left[\varepsilon_{2 r+2},\left[\varepsilon_{2 s+2}, \varepsilon_{2 t+2}\right]\right]$ with $r, s, t \geqslant 1$.

Proof. By Proposition 2.4, since $D \in \Theta^{3} \mathscr{E}$, there exists a polynomial $Q \in \operatorname{Lie}[a, b]$ such that $D(a)=[a, Q]$. By (iii) of Theorem 3.1, this means that we have the following relation between the depth 3 moulds $\operatorname{ma}(D(a))$ and $\operatorname{ma}(Q)$ :

$$
-\left(u_{1}+u_{2}+u_{3}\right) \operatorname{ma}(Q)=\operatorname{ma}(D(a)) \text {. }
$$

Thus ma $(D(a))$ is a polynomial divisible by the factor $\left(u_{1}+u_{2}+u_{3}\right)$, and since the mould ma $(D(a))$ is push-invariant by Lemma 2.3 and (ii) of Theorem 3.1, it is also divisible by $u_{1}, u_{2}$ and $u_{3}$. Thus in fact $\operatorname{ma}(D(a))$ is divisible by $Д_{3}$, so $\Psi(D)=$ $\mathrm{Da}(D(a))$ is a polynomial-valued mould in $\mathcal{U}$ concentrated in depth 3 . Now, we saw that $\mathcal{U}$ is contained in $\mathrm{ARI}_{\text {al } / \mathrm{al}}^{\mathrm{pol}}$, i.e. every mould in $\mathcal{U}$ is bialternal. In particular, the depth 3 polynomial mould $\frac{\text { al }}{\Psi}(\bar{D}) \in \mathcal{U}$ is bialternal. By Theorem 4.1, we can write it as

$$
\Psi(D)\left(u_{1}, u_{2}, u_{3}\right)=\sum_{r, s, t \geqslant 1} c_{r s t} \operatorname{ari}\left(U_{2 r}, \operatorname{ari}\left(U_{2 s}, U_{2 t}\right)\right) .
$$

Then since $\Psi\left(\varepsilon_{2 i}\right)=U_{2 i-2}$ and $\Psi: \mathscr{E} \rightarrow \mathcal{U}$ is a Lie algebra isomorphism by Corollary 3.6, if we set

$$
H=\sum_{r, s, t \geqslant 1} c_{r s t}\left[\varepsilon_{2 r+2},\left[\varepsilon_{2 s+2}, \varepsilon_{2 t+2}\right]\right]
$$

we must have $\Psi(D)=\Psi(H)$, so by the injectivity of $\Psi$, we have $D=H$, proving the theorem.

\section{Appendix A. Proof of Theorem 3.3}

This appendix is devoted to the proof of Theorem 3.3, which makes essential use of the swap operator and some of the basic notions of Ecalle's theory of moulds, in particular the ari-bracket.

Definitions. If we break the tuple $w=\left(u_{1}, \ldots, u_{r}\right)$ into three parts

$$
a=\left(u_{1}, \ldots, u_{k}\right), \quad b=\left(u_{k+1}, \ldots, u_{\ell}\right), \quad c=\left(u_{\ell+1}, \ldots, u_{r}\right),
$$

we write

$$
\begin{cases}a\left\lceil c=\left(u_{1}, \ldots, u_{k}, u_{k+1}+\cdots+u_{\ell}+u_{\ell+1}, u_{\ell+2}, \ldots, u_{r}\right)\right. & \text { if } c \neq \varnothing \\ a\rceil c=\left(u_{1}, \ldots, u_{k}+u_{k+1}+\cdots+u_{\ell}, u_{\ell+1}, \ldots, u_{r}\right) & \text { if } a \neq \varnothing\end{cases}
$$

If $A, B \in \mathrm{ARI}$, we define the operator $\operatorname{arit}(B)$ acting on $A$ by

$$
(\operatorname{arit}(B) \cdot A)\left(u_{1}, \ldots, u_{r}\right)=\sum_{0 \leqslant k<\ell<r} A\left(a\lceil c) B(b)-\sum_{1 \leqslant k<\ell \leqslant r} A(a\rceil c\right) B(b) .
$$

If $A, B \in \overline{\mathrm{ARI}}$ and the tuple $\left(v_{1}, \ldots, v_{r}\right)$ breaks into pieces

$$
a=\left(v_{1}, \ldots, v_{k}\right), \quad b=\left(v_{k+1}, \ldots, v_{\ell}\right), \quad c=\left(v_{\ell+1}, \ldots, v_{r}\right),
$$

then setting

$$
\begin{cases}b\rfloor=\left(u_{k+1}-u_{\ell+1}, u_{k+2}-u_{\ell+1}, \ldots, u_{\ell}-u_{\ell+1}\right) & \text { if } c \neq \varnothing, \text { otherwise } b\rfloor=b \\ \left\lfloor b=\left(u_{k+1}-u_{k}, u_{k+2}-u_{k}, \ldots, u_{\ell}-u_{k}\right)\right. & \text { if } a \neq \varnothing, \text { otherwise }\lfloor b=b\end{cases}
$$


we let

$$
\left.(\operatorname{arit}(B) \cdot A)\left(v_{1}, \ldots, v_{r}\right)=\sum_{0 \leqslant k<\ell<r} A(a c) B(b\rfloor\right)-\sum_{1 \leqslant k<\ell \leqslant r} A(a c) B(\lfloor b) .
$$

For two moulds $A$ and $B$, let $\operatorname{mu}(A, B)$ be the product defined by

$$
\operatorname{mu}(A, B)\left(u_{1}, \ldots, u_{r}\right)=\sum_{i=0}^{r} A\left(u_{1}, \ldots, u_{i}\right) B\left(u_{i+1}, \ldots, u_{r}\right) .
$$

When $A=\operatorname{ma}(F)$ and $B=\operatorname{ma}(G)$ for polynomials $F, G$ in $a, b$, the multiplication mu coincides with ordinary multiplication of polynomials: $\operatorname{mu}(\operatorname{ma}(F), \operatorname{ma}(G))=$ $\operatorname{ma}(F G)$ (cf. $[22,(3.2 .13)])$. Let $\operatorname{lu}(A, B)=\operatorname{mu}(A, B)-\operatorname{mu}(B, A)$, so lu is the corresponding Lie bracket. For $A, B \in \mathrm{ARI}$ or $\overline{\mathrm{ARI}}$, we set

$$
\operatorname{ari}(A, B)=\operatorname{arit}(B) \cdot A-\operatorname{arit}(A) \cdot B+\operatorname{lu}(A, B) .
$$

Recall that $Д$ is the mould in the $u_{i}$ 's defined by

$$
\text { Д }\left(u_{1}, \ldots, u_{r}\right)=u_{1} \cdots u_{r}\left(u_{1}+\cdots+u_{r}\right) .
$$

Let $Д_{v}=\operatorname{swap}($ Д), explicitly

$$
\text { Д }_{v}\left(v_{1}, \ldots, v_{r}\right)=v_{1}\left(v_{1}-v_{2}\right) \cdots\left(v_{r-1}-v_{r}\right) v_{r} .
$$

For any mould $A \in \overline{\mathrm{ARI}}$, let $\check{A}$ be the mould defined by

$$
\check{A}\left(v_{1}, \ldots, v_{r}\right)=Д_{v}\left(v_{1}, \ldots, v_{r}\right) A\left(v_{1}, \ldots, v_{r}\right) .
$$

Let $\overline{\mathrm{ARI}}_{\mathrm{al}}^{\text {sing }}$ denote the space of alternal rational-valued moulds $A$ in the variables $v_{i}$ such that $\check{A}$ is polynomial-valued.

The heart of the proof of Theorem 3.3 [1, lemme 4.40] consists in the following proposition.

Proposition A.1. The space $\overline{\mathrm{ARI}}_{\mathrm{al}}^{\mathrm{sing}}$ is a Lie algebra under the ari-bracket.

We first need a lemma.

Lemma A.2. Let $M$ be an element of $\overline{\mathrm{ARI}}_{\mathrm{al}}^{\text {sing }}$. Then the mould $\check{M}$ satisfies the relation

$$
\check{M}\left(0, v_{2}, \ldots, v_{r}\right)=\check{M}\left(v_{2}, \ldots, v_{r}, 0\right) \text {. }
$$

Proof of Lemma A.2. Let us write the simplest of the alternality relations, which gives

$$
\begin{aligned}
0= & \sum_{w \in \operatorname{sha}\left(v_{1}, v_{2} \cdots v_{r}\right)} M(w) \\
= & \sum_{i=0}^{r} M\left(v_{2}, \ldots, v_{i}, v_{1}, v_{i+1}, \ldots, v_{r}\right) \\
= & \sum_{i=1}^{r-1} \frac{\check{M}\left(v_{2}, \ldots, v_{i}, v_{1}, v_{i+1}, \ldots, v_{r}\right)}{v_{2}\left(v_{2}-\cdots\right) \cdots\left(\cdots-v_{r}\right) v_{r}}+ \\
& +\frac{\check{M}\left(v_{1}, \ldots, v_{r}\right)}{v_{1}\left(v_{1}-v_{2}\right) \cdots\left(v_{r-1}-v_{r}\right) v_{r}}+ \\
& +\frac{\check{M}\left(v_{2}, \ldots, v_{r}, v_{1}\right)}{v_{2}\left(v_{2}-v_{3}\right) \cdots\left(v_{r-1}-v_{r}\right)\left(v_{r}-v_{1}\right) v_{1}} .
\end{aligned}
$$


Hence, multiplying by $v_{1}$ and evaluating at $v_{1}=0$,

$$
0=\frac{\check{M}\left(0, v_{2}, \ldots, v_{r}\right)}{\left(-v_{2}\right)\left(v_{2}-v_{3}\right) \cdots\left(v_{r-1}-v_{r}\right) v_{r}}+\frac{\check{M}\left(v_{2}, \ldots, v_{r}, 0\right)}{v_{2}\left(v_{2}-v_{3}\right) \cdots\left(v_{r-1}-v_{r}\right) v_{r}},
$$

which is well-defined since both numerators at play are polynomials by the hypothesis on $M$. The result follows immediately.

Proof of Proposition A.1. Let $M, N \in \overline{\mathrm{ARI}}_{\mathrm{al}}^{\text {sing }}$. By linearity, we may assume that $M$ and $N$ are respectively concentrated in depths $r \geqslant 1$ and $s \geqslant 1$. Now, alternality is preserved by the ari-bracket (cf. [19, Theorem 3.1] for a complete proof), so it only remains to show that $\operatorname{ari}(M, N)$ is an element of $\overline{\mathrm{ARI}}^{\text {sing }}$.

The essential step of the proof consists in determining the poles of $\operatorname{arit}(M) \cdot N$ by reducing their values modulo the subspace of polynomials in the $v_{i}$ 's. We will use the simplifying notation $\beth_{v}\left(v_{1}, \ldots, v_{r}\right)=\beth_{r}(v)$. The fact that $M$ is concentrated in depth $r$ and $N$ in depth $s$ also simplifies the defining formula for $\operatorname{arit}(M) \cdot N$, as follows:

$$
\begin{aligned}
\left.(\operatorname{arit}(M) \cdot N)\left(v_{1} \ldots v_{r+s}\right)=\sum_{0 \leqslant i<s} N\left(v_{1} \cdots v_{i} v_{i+r+1} \cdots v_{r+s}\right) M\left(v_{i+1} \cdots v_{i+r}\right\rfloor\right)- \\
\quad \sum_{0<i \leqslant s} N\left(v_{1} \cdots v_{i} v_{i+r+1} \cdots v_{r+s}\right) M\left(\left\lfloor v_{i+1} \cdots v_{i+r}\right)\right. \\
=N\left(v_{r+1} \cdots v_{r+s}\right) M\left(v_{1}-v_{r+1}, \ldots, v_{r}-v_{r+1}\right) \\
+\sum_{i=1}^{s-1} N\left(v_{1} \cdots v_{i} v_{i+r+1} \cdots v_{r+s}\right) . \\
\quad\left(M\left(v_{i+1}-v_{i+r+1}, \ldots, v_{i+r}-v_{i+r+1}\right)-M\left(v_{i+1}-v_{i}, \ldots, v_{i+r}-v_{i}\right)\right) \\
\quad-N\left(v_{1} \cdots v_{s}\right) M\left(v_{s+1}-v_{s}, \ldots v_{r+s}-v_{s}\right) .
\end{aligned}
$$

Consequently,

$$
\begin{aligned}
& Д_{r+s}(v)(\operatorname{arit}(M) \cdot N)\left(v_{1}, \ldots, v_{r+s}\right)= \\
& \quad \frac{v_{1}}{v_{r+1}\left(v_{1}-v_{r+1}\right)} \check{N}\left(v_{r+1} \cdots v_{r+s}\right) \check{M}\left(v_{1}-v_{r+1}, \ldots, v_{r}-v_{r+1}\right)+\sum_{i=1}^{s-1} Д_{r+s}(v) S_{i} \\
& \quad+\frac{v_{r+s}}{v_{s}\left(v_{r+s}-v_{s}\right)} \check{N}\left(v_{1} \cdots v_{s}\right) \check{M}\left(v_{s+1}-v_{s}, \ldots, v_{r+s}-v_{s}\right)
\end{aligned}
$$

with

$$
\begin{aligned}
\beth_{r+s}(v) S_{i}= & \frac{\left(v_{i}-v_{i+1}\right) \cdots\left(v_{i+r}-v_{i+r+1}\right)}{v_{i}-v_{i+r+1}} \check{N}\left(v_{1} \cdots v_{i} v_{i+r+1} \cdots v_{r+s}\right) . \\
& \left(M\left(v_{i+1}-v_{i+r+1}, \ldots, v_{i+r}-v_{i+r+1}\right)-M\left(v_{i+1}-v_{i}, \ldots, v_{i+r}-v_{i}\right)\right) \\
= & \frac{\left(v_{i}-v_{i+1}\right) \cdots\left(v_{i+r}-v_{i+r+1}\right)}{v_{i}-v_{i+r+1}} \check{N}\left(v_{1} \cdots v_{i} v_{i+r+1} \cdots v_{r+s}\right) . \\
& \left(\frac{\check{M}\left(v_{i+1}-v_{i+r+1}, \ldots, v_{i+r}-v_{i+r+1}\right)}{\left(v_{i+1}-v_{i+r+1}\right)\left(v_{i+1}-v_{i+2}\right) \cdots\left(v_{i+r-1}-v_{i+r}\right)\left(v_{i+r}-v_{i+r+1}\right)}-\right. \\
& \left.\frac{\check{M}\left(v_{i+1}-v_{i}, \ldots, v_{i+r}-v_{i}\right)}{\left(v_{i+1}-v_{i}\right)\left(v_{i+1}-v_{i+2}\right) \cdots\left(v_{i+r-1}-v_{i+r}\right)\left(v_{i+r}-v_{i}\right)}\right)
\end{aligned}
$$


or, equivalently,

$$
\begin{aligned}
\beth_{r+s}(v) S_{i}= & \frac{1}{v_{i}-v_{i+r+1}} \check{N}\left(v_{1} \cdots v_{i} v_{i+r+1} \cdots v_{r+s}\right) . \\
& \left(\frac{v_{i}-v_{i+1}}{v_{i+1}-v_{i+r+1}} \check{M}\left(v_{i+1}-v_{i+r+1}, \ldots, v_{i+r}-v_{i+r+1}\right)\right. \\
& \left.\quad+\frac{v_{i+r}-v_{i+r+1}}{v_{i+r}-v_{i}} \check{M}\left(v_{i+1}-v_{i}, \ldots, v_{i+r}-v_{i}\right)\right) .
\end{aligned}
$$

In light of equalities (A.2) and (A.3), there are three types of poles which can occur in the rational function $\beth_{r+s}(v)(\operatorname{arit}(M) \cdot N)$ :

1) the poles $\frac{1}{v_{r+1}}$ and $\frac{1}{v_{s}}$, which come from only one term and thus do not immediately cancel out;

2) the poles of the form $\frac{1}{v_{i}-v_{i+r+1}}$

3) the poles of the form $\frac{1}{v_{i}-v_{i+r}}$.

Let us deal first with case 2 . The corresponding pole $\frac{1}{v_{i}-v_{i+r+1}}$ can only appear in the term $Д_{r+s}(v) S_{i}$; we then want to check that this pole is compensated by the corresponding difference of the $\check{M}$ 's. Let us compute this difference for $v_{i}=v_{i+r+1}=$ $u_{i}=u_{i+r+1}=a$; it gives

$$
\frac{a-v_{i+1}}{v_{i+1}-a} \check{M}\left(v_{i+1}-a, \ldots, v_{i+r}-a\right)+\frac{v_{i+r}-a}{v_{i+r}-a} \check{M}\left(v_{i+1}-a, \ldots, v_{i+r}-a\right)
$$

which is indeed zero, hence the compensation.

For case 3 , we need to distinguish three sub-cases, according to whether $i=1$, or $i=s$, or $i$ belongs to $\llbracket 2, s-1 \rrbracket$.

a) $i=1$ : the pole is multiplied by

$$
\begin{aligned}
\frac{v_{1}}{v_{r+1}} \check{N}\left(v_{r+1} v_{r+2} \cdots v_{r+s}\right) \check{M}\left(v_{1}-v_{r+1}, \ldots, v_{r}-v_{r+1}\right)- \\
\quad-\frac{1}{v_{1}-v_{r+2}} \check{N}\left(v_{1} v_{r+2} \cdots v_{r+s}\right) \cdot\left(v_{r+1}-v_{r+2}\right) \check{M}\left(v_{2}-v_{1}, \ldots, v_{r+1}-v_{1}\right)
\end{aligned}
$$

which if $v_{1}=v_{r+1}=a$ gives

$$
\begin{aligned}
& \frac{a}{a} \check{N}\left(a, v_{r+2}, \ldots, v_{r+s}\right) \check{M}\left(a-a, v_{2}-a, \ldots, v_{r}-a\right)- \\
& \quad-\frac{1}{a-v_{r+2}} \check{N}\left(a, v_{r+2}, \cdots, v_{r+s}\right) \cdot\left(a-v_{r+2}\right) \check{M}\left(v_{2}-a, \ldots, v_{r}-a, a-a\right) \\
& =\check{N}\left(a, v_{r+2}, \ldots, v_{r+s}\right) \check{M}\left(0, v_{2}-a, \ldots, v_{r}-a\right)- \\
& \quad-\check{N}\left(a, v_{r+2}, \cdots, v_{r+s}\right) \check{M}\left(v_{2}-a, \ldots, v_{r}-a, 0\right)
\end{aligned}
$$

which is zero thanks to Lemma A.2.

b) $i=s$ : the pole is multiplied by

$$
\begin{aligned}
& -\frac{v_{r+s}}{v_{s}} \check{N}\left(v_{1} \cdots v_{s}\right) \check{M}\left(v_{s+1}-v_{s}, \ldots, v_{r+s}-v_{s}\right)+ \\
& +\frac{1}{v_{s-1}-v_{r+s}} \check{N}\left(v_{1}, \ldots, v_{s-1}, v_{r+s}\right) \cdot\left(v_{s-1}-v_{s}\right) \check{M}\left(v_{s}-v_{r+s}, \ldots, v_{r+s-1}-v_{r+s}\right)
\end{aligned}
$$

which if $v_{s}=v_{r+s}=a$ gives

$$
\begin{aligned}
& -\frac{a}{a} \check{N}\left(v_{1}, \ldots, v_{s-1}, a\right) \check{M}\left(v_{s+1}-a, \ldots, v_{r+s-1}-a, a-a\right)+ \\
& \quad+\frac{1}{v_{s-1}-a} \check{N}\left(v_{1}, \ldots, v_{s-1}, a\right) \cdot\left(v_{s-1}-a\right) \check{M}\left(a-a, v_{s+1}-a, \ldots, v_{r+s-1}-a\right) \\
& =-\check{N}\left(v_{1}, \ldots, v_{s-1}, a\right) \check{M}\left(v_{s+1}-a, \ldots, v_{r+s-1}-a, 0\right)+ \\
& \quad+\check{N}\left(v_{1}, \ldots, v_{s-1}, a\right) \check{M}\left(0, v_{s+1}-a, \ldots, v_{r+s-1}-a\right)
\end{aligned}
$$

which is zero for the same reason as above. 
c) $i \in \llbracket 2, s-1 \rrbracket$ : the pole $\frac{1}{v_{i}-v_{i+r}}$ comes from $Д_{r+s}(v)\left(S_{i-1}-S_{i}\right)$, and is multiplied by

$$
\begin{array}{r}
\frac{v_{i-1}-v_{i}}{v_{i-1}-v_{i+r}} \check{N}\left(v_{1}, \ldots, v_{i-1}, v_{i+r}, \cdots, v_{r+s}\right) \cdot \check{M}\left(v_{i}-v_{i+r}, \ldots, v_{i-1+r}-v_{i+r}\right)- \\
\quad-\frac{v_{i+r}-v_{i+r+1}}{v_{i}-v_{i+r+1}} \check{N}\left(v_{1}, \ldots, v_{i}, v_{i+r+1}, \ldots, v_{r+s}\right) \cdot \check{M}\left(v_{i+1}-v_{i}, \ldots, v_{i+r}-v_{i}\right)
\end{array}
$$

which if $v_{i}=v_{i+r}=a$ gives

$$
\begin{aligned}
& \check{N}\left(v_{1}, \ldots, v_{i-1}, a, v_{i+r+1}, \cdots, v_{r+s}\right) \cdot \check{M}\left(a-a, v_{i+1}-a, \ldots, v_{i-1+r}-a\right)- \\
& \quad-\check{N}\left(v_{1}, \ldots, v_{i-1}, a, v_{i+r+1}, \ldots, v_{r+s}\right) \cdot \check{M}\left(v_{i+1}-a, \ldots, v_{i+r-1}-a, a-a\right) \\
& =\check{N}\left(v_{1}, \ldots, v_{i-1}, a, v_{i+r+1}, \cdots, v_{r+s}\right) \cdot \check{M}\left(0, v_{i+1}-a, \ldots, v_{i-1+r}-a\right)- \\
& \quad-\check{N}\left(v_{1}, \ldots, v_{i-1}, a, v_{i+r+1}, \ldots, v_{r+s}\right) \cdot \check{M}\left(v_{i+1}-a, \ldots, v_{i+r-1}-a, 0\right)
\end{aligned}
$$

which is zero again.

In consequence, the only remaining poles in $\beth_{r+s}(v)(\operatorname{arit}(M) \cdot N)$ are those in $\frac{1}{v_{r+1}}$ and $\frac{1}{v_{s}}$ from case 1 , and more precisely we can write

$$
\begin{aligned}
Д_{r+s}(v) & (\operatorname{arit}(M) \cdot N)\left(v_{1}, \ldots, v_{r+s}\right) \equiv \\
& \frac{v_{1}}{v_{r+1}\left(v_{1}-v_{r+1}\right)} \check{N}\left(v_{r+1}, \ldots, v_{r+s}\right) \check{M}\left(v_{1}-v_{r+1}, \ldots, v_{r}-v_{r+1}\right) \\
& +\frac{v_{r+s}}{v_{s}\left(v_{r+s}-v_{s}\right)} \check{N}\left(v_{1}, \ldots, v_{s}\right) \check{M}\left(v_{s+1}-v_{s}, \ldots v_{r+s}-v_{s}\right) \quad(\bmod \mathbb{Q}[\underline{v}]) .
\end{aligned}
$$

It remains to show that the above expression cancels in the bracket $\operatorname{ari}(M, N)$, using the definition by equation (A.1). The possible poles for the rational function $\beth_{r+s}(v) \operatorname{ari}(M, N)$ come from the sum

$$
\begin{aligned}
& \frac{v_{1}}{v_{s+1}\left(v_{1}-v_{s+1}\right)} \check{M}\left(v_{s+1}, \ldots, v_{s+r}\right) \check{N}\left(v_{1}-v_{s+1}, \ldots, v_{s}-v_{s+1}\right)+ \\
& \quad+\frac{v_{s+r}}{v_{r}\left(v_{s+r}-v_{r}\right)} \check{M}\left(v_{1}, \ldots, v_{r}\right) \check{N}\left(v_{r+1}-v_{r}, \ldots, v_{s+r}-v_{r}\right)- \\
& \quad-\frac{v_{1}}{v_{r+1}\left(v_{1}-v_{r+1}\right)} \check{N}\left(v_{r+1}, \ldots, v_{r+s}\right) \check{M}\left(v_{1}-v_{r+1}, \ldots, v_{r}-v_{r+1}\right)+ \\
& \quad+\frac{v_{r+s}}{v_{s}\left(v_{r+s}-v_{s}\right)} \check{N}\left(v_{1}, \ldots, v_{s}\right) \check{M}\left(v_{s+1}-v_{s}, \ldots v_{r+s}-v_{s}\right)+ \\
& \quad+\frac{v_{r}-v_{r+1}}{v_{r} v_{r+1}} \check{M}\left(v_{1}, \ldots, v_{r}\right) \check{N}\left(v_{r+1}, \ldots, v_{r+s}\right)- \\
& \quad-\frac{v_{s}-v_{s+1}}{v_{s} v_{s+1}} \check{N}\left(v_{1}, \ldots, v_{s}\right) \check{M}\left(v_{s+1}, \ldots, v_{r+s}\right)
\end{aligned}
$$

paying attention to the exchange of $r$ and $s$ when we switch $M$ and $N$. The discussion on page 12 shows that it suffices to check that the poles $\frac{1}{v_{r}}$ and $\frac{1}{v_{s}}, \frac{1}{v_{r+1}}$ and $\frac{1}{v_{s+1}}$ cancel out.

Let us note that the alternality of $\operatorname{ari}(M, N)$ together with the basic fact that any alternal mould $M$ satisfies $M\left(u_{1}, \ldots, u_{r}\right)=(-1)^{r-1} M\left(u_{r}, \ldots, u_{1}\right)$ [22, Lemma 2.5.3] imply that it is enough to deal with the case of $\frac{1}{v_{r}}$ and $\frac{1}{v_{s}}$, the other two being deduced from them by applying the involution corresponding to the symmetry around $\frac{r+s+1}{2}$. And the pole $\frac{1}{v_{s}}$ is deduced from $\frac{1}{v_{r}}$ since the total expression for $\operatorname{ari}(M, N)$ is antisymmetric in $M$ and $N$, and thus in $r$ and $s$.

The only case left to check is that of the pole $\frac{1}{v_{r}}$. The corresponding factor is

$$
\begin{gathered}
\frac{v_{s+r}}{v_{s+r}-v_{r}} \check{M}\left(v_{1}, \ldots, v_{r}\right) \check{N}\left(v_{r+1}-v_{r}, \ldots, v_{s+r}-v_{r}\right)+ \\
\quad+\frac{v_{r}-v_{r+1}}{v_{r+1}} \check{M}\left(v_{1}, \ldots, v_{r}\right) \check{N}\left(v_{r+1}, \ldots, v_{r+s}\right)
\end{gathered}
$$

which is clearly zero when $v_{r}=0$. This concludes the proof of Proposition A.1. 
We can now complete the proof of Theorem 3.3. We use the following elementary result of mould theory [22, Lemma 2.4.1],

$$
\operatorname{ari}(\operatorname{swap}(A), \operatorname{swap}(B))=\operatorname{swap}(\operatorname{ari}(A, B)) \quad \forall A, B \in \mathrm{ARI}_{\text {push }} .
$$

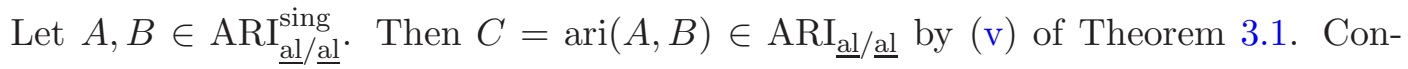
sider the mould $\operatorname{swap}(C) \in \overline{\mathrm{ARI}}$. Again by $(\mathrm{v})$ of Theorem 3.1, $\mathrm{ARI}_{\mathrm{al} / \mathrm{al}}$ is contained in $\mathrm{ARI}_{\text {push }}$, so the moulds $A, B$ and $C$ are all push-invariant. Thus (A.4) holds, i.e. $\operatorname{swap}(C)=\operatorname{ari}(\operatorname{swap}(A), \operatorname{swap}(B))$.

$\operatorname{Both} \operatorname{swap}(A)$ and $\operatorname{swap}(B)$ lie in $\overline{\mathrm{ARI}}_{\text {al }}^{\text {sing }}$ by definition. Thus by Proposition A.1, we also have $\operatorname{swap}(C) \in \overline{\mathrm{ARI}}_{\mathrm{al}}^{\text {sing }}$ and thus $C \in \mathrm{ARI}^{\text {sing }}$. But we also have $C=\operatorname{ari}(A, B) \in$ $\mathrm{ARI}_{\underline{a l} / \underline{a l}}$, so $C \in \mathrm{ARI}_{\underline{a l} / \underline{\mathrm{a}}}^{\mathrm{sing}}$, which concludes the proof of Theorem 3.3.

\section{Appendix B. Proof of Theorem 3.5}

This section is devoted to the proof of Theorem 3.5, which is stated as Proposition B.2 below.

We use the notation and terminology of section 3, with one further definition: for any polynomial $F \in \operatorname{Lie}[a, b]$ of homogeneous depth $r$, we define da $(F)$ to be the mould given by

$$
\mathrm{da}(F)\left(u_{1}, \ldots, u_{r}\right)=\frac{\operatorname{ma}(F)}{u_{1} \cdots u_{r}} .
$$

As we did for $\mathrm{Da}(F)$, if $F=\sum_{r} F^{r}$ is any Lie polynomial broken up into its depthgraded parts, we define $\mathrm{da}(F)=\sum_{r} \mathrm{da}\left(F^{r}\right)$.

Let $\operatorname{lu}(A, B)$ be as in the beginning of Appendix A. For any polynomial $U$, let $\operatorname{Darit}_{U}$ be the operator on moulds defined by

$$
\operatorname{Darit}_{U} \cdot A=-\operatorname{arit}(\operatorname{Da}(U)) \cdot A-\mathrm{lu}(A, \operatorname{Da}(U)) .
$$

Proposition B.1. Let $U$ be a push-invariant polynomial in $\operatorname{Lie}[a, b]$, and let $D_{U}$ be the associated derivation. Then for any Lie polynomial $F$ in $a$ and $b$, we have

$$
-\operatorname{da}\left(D_{U}(F)\right)=\operatorname{Darit}_{U} \cdot \operatorname{da}(F) .
$$

Proof. By additivity, we may assume that $U$ is of homogeneous depth $r$ and $F$ is of homogeneous depth $s$. We will use induction on the depth of $F$ to prove the proposition; the only difficult part is the base case $s=1$.

So assume that $F$ is of depth 1, i.e. $F=C_{n}=\operatorname{ad}(a)^{n-1}(b)$. Here, since $D_{U}([a, b])$ is zero, we have the explicit formula

$$
D_{U}(F)=\sum_{i=0}^{n-2} \operatorname{ad}(a)^{i} \operatorname{ad}(U) \operatorname{ad}(a)^{n-i-2}(b)=\sum_{i=0}^{n-2} \operatorname{ad}(a)^{i}\left(U C_{n-i-1}-C_{n-i-1} U\right) .
$$

Applying ma to both sides of this formula, and using the facts that ma $\left(C_{n-i-1}\right)$ is equal to $(-1)^{n-i-2} u_{1}^{n-i-2}$ and that for a Lie polynomial $P$ of homogeneous depth $r$ we have

$$
\operatorname{ma}([a, P])=-\left(u_{1}+\cdots+u_{r}\right) \operatorname{ma}(P)
$$

by (iii) of Theorem 3.1, and using the identity

$$
\begin{aligned}
\operatorname{ma}\left(U C_{n-i-1}-C_{n-i-1} U\right)= & (-1)^{n-i-2} \operatorname{ma}(U)\left(u_{1}, \ldots, u_{r}\right) u_{r+1}^{n-i-2} \\
& -(-1)^{n-i-2} u_{1}^{n-i-2} \operatorname{ma}(U)\left(u_{2}, \ldots, u_{r+1}\right),
\end{aligned}
$$


we find that

$$
\begin{aligned}
\operatorname{ma}\left(D_{U}(F)\right)= & \sum_{i=0}^{n-3}(-1)^{n-1}\left(u_{1}+\cdots+u_{r+1}\right)^{i} . \\
& \left(\operatorname{ma}(U)\left(u_{1}, \ldots, u_{r}\right) u_{r+1}^{n-i-2}-u_{1}^{n-i-2} \operatorname{ma}(U)\left(u_{2}, \ldots, u_{r+1}\right)\right),
\end{aligned}
$$

so, with a little rewriting of indices, we find that the left-hand side of (B.1) equals

$$
\begin{aligned}
-\mathrm{da}\left(D_{U}(F)\right)= & (-1)^{n-1} \frac{\operatorname{ma}(U)\left(u_{2}, \ldots, u_{r+1}\right)}{\beth_{r+1}} \sum_{i=1}^{n-2}\left(u_{1}+\cdots+u_{r+1}\right)^{i} u_{1}^{n-i-1} \\
& -(-1)^{n-1} \frac{\operatorname{ma}(U)\left(u_{1}, \ldots, u_{r}\right)}{\beth_{r+1}} \sum_{i=1}^{n-2}\left(u_{1}+\cdots+u_{r+1}\right)^{i} u_{r+1}^{n-i-1},
\end{aligned}
$$

or, adding up the sums to obtain a closed expression,

$$
\begin{gathered}
-\mathrm{da}\left(D_{U}(F)\right)=(-1)^{n-1} \frac{\mathrm{ma}(U)\left(u_{2}, \ldots, u_{r+1}\right)}{\beth_{r+1}}\left(\frac{\left(u_{1}+\cdots+u_{r+1}\right)^{n-1} u_{1}-u_{1}^{n-1}\left(u_{1}+\cdots+u_{r+1}\right)}{\left(u_{2}+\cdots+u_{r+1}\right)}\right) \\
-(-1)^{n-1} \frac{\mathrm{ma}(U)\left(u_{1}, \ldots, u_{r}\right)}{Д_{r+1}}\left(\frac{\left(u_{1}+\cdots+u_{r+1}\right)^{n-1} u_{r+1}-u_{r+1}^{n-1}\left(u_{1}+\cdots+u_{r+1}\right)}{\left(u_{1}+\cdots+u_{r}\right)}\right)
\end{gathered}
$$

Let us now compute the right-hand side of (B.1). We have $\operatorname{ma}(F)=(-1)^{n} u_{1}^{n-1}$, so $\operatorname{da}(F)=(-1)^{n} u_{1}^{n-2} ;$ thus

$$
\mathrm{lu}(\mathrm{da}(F), \operatorname{Da}(U))=(-1)^{n}\left(\frac{u_{1}^{n-2} \operatorname{ma}(U)\left(u_{2}, \ldots, u_{r+1}\right)}{u_{2} \cdots u_{r+1}\left(u_{2}+\cdots+u_{r+1}\right)}\right)-(-1)^{n}\left(\frac{\operatorname{ma}(U)\left(u_{1}, \ldots, u_{r}\right) u_{r+1}^{n-2}}{u_{1} \cdots u_{r}\left(u_{1}+\cdots+u_{r}\right)}\right) .
$$

Also, we have

$$
\begin{aligned}
\operatorname{arit} & \operatorname{Da}(U)) \cdot \operatorname{da}(F)=\sum_{w=a b c, c \neq \varnothing} \operatorname{da}(F)\left(a\lceil c) \operatorname{Da}(U)(b)-\sum_{w=a b c, a \neq \varnothing} \operatorname{da}(F)(a\rceil c\right) \operatorname{Da}(U)(b) \\
= & (-1)^{n}\left(u_{1}+\cdots+u_{r+1}\right)^{n-2} \operatorname{Da}(U)\left(u_{1}, \ldots, u_{r}\right)- \\
& \quad-(-1)^{n}\left(u_{1}+\cdots+u_{r+1}\right)^{n-2} \operatorname{Da}(U)\left(u_{2}, \ldots, u_{r+1}\right) \\
= & (-1)^{n}\left(u_{1}+\cdots+u_{r+1}\right)^{n-2}\left(\frac{\operatorname{ma}(U)\left(u_{1}, \ldots, u_{r}\right)}{u_{1} \cdots u_{r}\left(u_{1}+\cdots+u_{r}\right)}-\frac{\operatorname{ma}(U)\left(u_{2}, \ldots, u_{r+1}\right)}{u_{2} \cdots u_{r+1}\left(u_{2}+\cdots+u_{r+1}\right)}\right)
\end{aligned}
$$

since da $(F)$ is a depth 1 mould and therefore the only possible decomposition $w=a b c$ with $c \neq \varnothing$ is $a=\varnothing, b=\left(u_{1}, \ldots, u_{r}\right)$ and $c=u_{r+1}$, and the only possible decomposition with $a \neq \varnothing$ is $a=u_{1}, b=\left(u_{2}, \ldots, u_{r}\right)$ and $c=\varnothing$. We add (B.3) and (B.4) to get the right-hand side of (B.1), obtaining

$$
\begin{gathered}
\operatorname{Darit}_{U} \cdot \operatorname{da}(F)=(-1)^{n} \frac{\operatorname{ma}(U)\left(u_{1}, \ldots, u_{r}\right)}{u_{1} \cdots u_{r}\left(u_{1}+\cdots+u_{r}\right)}\left(\left(u_{1}+\cdots+u_{r+1}\right)^{n-2}-u_{r+1}^{n-2}\right) \\
+(-1)^{n} \frac{\operatorname{ma}(U)\left(u_{2}, \ldots, u_{r+1}\right)}{u_{2} \cdots u_{r+1}\left(u_{2}+\cdots+u_{r+1}\right)}\left(u_{1}^{n-2}-\left(u_{1}+\cdots+u_{r+1}\right)^{n-2}\right) \\
=(-1)^{n} \frac{\operatorname{ma}(U)\left(u_{1}, \ldots, u_{r}\right)}{\beth_{r+1}}\left(\left(u_{1}+\cdots+u_{r+1}\right)^{n-1} u_{r+1}-u_{r+1}^{n-1} \frac{\left(u_{1}+\cdots+u_{r+1}\right)}{\left(u_{1}+\cdots+u_{r}\right)}\right) \\
-(-1)^{n} \frac{\operatorname{ma}(U)\left(u_{2}, \ldots, u_{r+1}\right)}{\beth_{r+1}}\left(\frac{u_{1}\left(u_{1}+\cdots+u_{r+1}\right)^{n-1}-u_{1}^{n-1}\left(u_{1}+\cdots+u_{r+1}\right)}{\left(u_{2}+\cdots+u_{r+1}\right)}\right),
\end{gathered}
$$

which is equal to (B.2). This settles the base case where $F$ is of depth 1 . 
Now assume that (B.1) holds up to depth $s-1$, and let $F$ be a Lie polynomial of depth $s$. Then $F$ is a linear combination of Lie brackets, so by additivity, we may assume that $F$ is a single Lie bracket; thus $F=[G, H]$ for Lie brackets $G, H$ that are of depth $<s$. We saw in Appendix A that $\operatorname{ma}(F G)=\operatorname{mu}(\operatorname{ma}(F)$, ma $(G))$, so by definition of da we also have $\operatorname{mu}(\operatorname{da}(F), \operatorname{da}(G))=\operatorname{da}(F G)$. Furthermore Darit ${ }_{U}$ is a derivation for the lu-bracket since both $\operatorname{arit}(B)$ and $\mathrm{lu}(-, B)$ are - this is obvious for lu but difficult for arit, cf. [22, Prop. 2.2.1] —, so using the induction hypothesis for $G$ and $H$, we have

$$
\begin{aligned}
-\operatorname{da}\left(D_{U}(F)\right) & =-\operatorname{da}\left(\left[D_{U}(G), H\right]+\left[G, D_{U}(H)\right]\right) \\
& =-\operatorname{lu}\left(\operatorname{da}\left(D_{U}(G)\right), \operatorname{da}(H)\right)-\operatorname{lu}\left(\operatorname{da}(G), \operatorname{da}\left(D_{U}(H)\right)\right) \\
& \left.\left.=\operatorname{lu}\left(\operatorname{Darit}_{U}(\operatorname{da}(G))\right), \operatorname{da}(H)\right)+\operatorname{lu}\left(\operatorname{da}(G), \operatorname{Darit}_{U}(\operatorname{da}(H))\right)\right) \\
& \left.=\operatorname{Darit}_{U} \cdot \operatorname{lu}(\operatorname{da}(G)), \operatorname{da}(H)\right) \\
& =\operatorname{Darit}_{U} \cdot \operatorname{da}([G, H]) \\
& =\operatorname{Darit}_{U} \cdot \operatorname{da}(F) .
\end{aligned}
$$

This proves the proposition.

Proposition B.2. The map $\operatorname{Der}^{0} \operatorname{Lie}[a, b] \rightarrow$ ARI given by $D_{U} \mapsto \operatorname{Da}(U)$ is a Lie algebra morphism, i.e.

$$
\left[D_{U}, D_{V}\right] \mapsto \operatorname{ari}(\mathrm{Da}(U), \mathrm{Da}(V)) .
$$

Proof. It is easily seen that

$$
\operatorname{arit}(B) \circ \operatorname{arit}(A)-\operatorname{arit}(A) \circ \operatorname{arit}(B)=\operatorname{arit}(\operatorname{ari}(A, B)) .
$$

Using only this identity, the Jacobi relation and (B.1), we compute

$$
\begin{aligned}
& -\operatorname{da}\left(\left[D_{U}, D_{V}\right](F)\right)=-\operatorname{da}\left(D_{U}\left(D_{V}(F)\right)\right)+\operatorname{da}\left(\operatorname{ma}\left(D_{V}\left(D_{U}(F)\right)\right)\right. \\
& =\operatorname{arit}(\operatorname{Da}(U)) \cdot \operatorname{da}\left(D_{V}(F)\right)+\mathrm{lu}\left(\operatorname{da}\left(D_{V}(F)\right), \operatorname{Da}(U)\right) \\
& +\operatorname{arit}(\operatorname{Da}(V)) \cdot \operatorname{da}\left(D_{U}(F)\right)+\operatorname{lu}\left(\operatorname{da}\left(D_{U}(F)\right), \operatorname{Da}(V)\right) \\
& =-\operatorname{arit}(\operatorname{Da}(U)) \cdot(\operatorname{arit}(\operatorname{Da}(V)) \cdot \operatorname{da}(F)+\operatorname{lu}(\operatorname{da}(F), \operatorname{Da}(V))) \\
& -\mathrm{lu}(\operatorname{arit}(\operatorname{Da}(V)) \cdot \mathrm{da}(F), \operatorname{Da}(U))-\mathrm{lu}(\mathrm{lu}(\mathrm{da}(F), \operatorname{Da}(V)), \operatorname{Da}(U)) \\
& +\operatorname{arit}(\operatorname{Da}(V)) \cdot(\operatorname{arit}(\operatorname{Da}(U)) \cdot \operatorname{da}(F)+\mathrm{lu}(\operatorname{da}(F), \operatorname{Da}(U))) \\
& +\mathrm{lu}(\operatorname{arit}(\operatorname{Da}(U)) \cdot \operatorname{da}(F), \operatorname{Da}(V))-\mathrm{lu}(\mathrm{lu}(\mathrm{da}(F), \operatorname{Da}(U)), \operatorname{Da}(V)) \\
& =\operatorname{arit}(\operatorname{ari}(\operatorname{Da}(U), \operatorname{Da}(V))) \cdot \operatorname{da}(F) \quad(\text { by }(\mathrm{B} .7)) \\
& -\mathrm{lu}(\operatorname{da}(F), \operatorname{arit}(\operatorname{Da}(U)) \cdot \operatorname{Da}(V))+\mathrm{lu}(\operatorname{da}(F), \operatorname{arit}(\operatorname{Da}(V)) \cdot \operatorname{Da}(U)) \\
& -\operatorname{lu}(\operatorname{lu}(\operatorname{da}(F), \operatorname{Da}(V)), \operatorname{Da}(U))+\operatorname{lu}(\operatorname{lu}(\operatorname{da}(F), \operatorname{Da}(U)), \operatorname{Da}(V)) \\
& =\operatorname{arit}(\operatorname{ari}(\operatorname{Da}(U), \operatorname{Da}(V))) \cdot \operatorname{da}(F) \\
& -\mathrm{lu}(\operatorname{da}(F), \operatorname{arit}(\operatorname{Da}(U)) \cdot \operatorname{Da}(V))+\mathrm{lu}(\operatorname{da}(F), \operatorname{arit}(\operatorname{Da}(V)) \cdot \operatorname{Da}(U)) \\
& +\operatorname{lu}(\operatorname{da}(F), \operatorname{lu}(\operatorname{Da}(U), \operatorname{Da}(V))) \quad \text { (by Jacobi) } \\
& =\operatorname{arit}(\operatorname{ari}(\operatorname{Da}(U), \operatorname{Da}(V))) \cdot \operatorname{da}(F)
\end{aligned}
$$




$$
\begin{aligned}
& +\operatorname{lu}(\operatorname{da}(F),-\operatorname{arit}(\operatorname{Da}(U)) \cdot \operatorname{Da}(V)+\operatorname{arit}(\operatorname{Da}(V)) \cdot \operatorname{Da}(U)+\mathrm{lu}(\operatorname{Da}(U), \operatorname{Da}(V))) \\
= & \operatorname{arit}(\operatorname{ari}(\operatorname{Da}(U), \operatorname{Da}(V))) \cdot \operatorname{da}(F)+\mathrm{lu}(\operatorname{da}(F), \operatorname{ari}(\operatorname{Da}(U), \operatorname{Da}(V))) \\
= & \operatorname{Darit}_{W} \cdot \operatorname{da}(F),
\end{aligned}
$$

where $W$ is the polynomial such that $\mathrm{Da}(W)=\operatorname{ari}(\mathrm{Da}(U), \mathrm{Da}(V))$ (obtained by multiplying the right-hand mould by Д and inverting the injective map ma). However, the above calculation shows that

$$
\operatorname{Darit}_{W} \cdot \operatorname{da}(F)=-\mathrm{da}\left(\left[D_{U}, D_{V}\right](F)\right)=\operatorname{Darit}_{P} \cdot \operatorname{da}(F),
$$

where $P=D_{U}(V)-D_{V}(U)$, that is $D_{P}=\left[D_{U}, D_{V}\right]$. Thus the two derivations Darit ${ }_{W}$ and $\operatorname{Darit}_{P}$ take the same values on all elements $F$ of Lie $[a, b]$, so $W=P$, i.e. $\left[D_{U}, D_{V}\right]=$ $D_{P}=D_{W}$, so under the map $D_{U} \mapsto \operatorname{Da}(U)$ of (B.6), we have $\left[D_{U}, D_{V}\right]=D_{W} \mapsto$ $\operatorname{Da}(W)=\operatorname{ari}(\operatorname{Da}(U), \operatorname{Da}(V))$. This proves Proposition B.2.

\section{REFERENCES}

[1] S. Baumard, Aspects modulaires et elliptiques des relations entre multizêtas, Ph.D. thesis, June 2014, http://tel.archives-ouvertes.fr/tel-01017022.

[2] F. Brown, Mixed Tate motives over $\mathbb{Z}$, Annals of Math. (2) 175 (2012), no. 2, 949-976.

[3] - Depth-graded motivic multiple zeta values, preprint, 2013 (see www.ihes.fr/ brown).

[4] Zeta elements in depth 3 and the fundamental Lie algebra of a punctured elliptic curve, preprint, 2015 (see www.ihes.fr/ brown).

[5] - Anatomy of the motivic Lie algebra, online lecture notes, April 2013 (see www. ihes.fr/ brown).

[6] J. Ecalle, The flexion structure and dimorphy, in Asymptotics in Dynamics, Geometry and PDEs; Generalized Borel Summation, Publications of the Scuola Normale Superiore, vol. 12 (2011), no. 2, p. 27-211.

[7] B. Enriquez, Elliptic associators, Selecta Math. New Series 20 (2014), 491-584.

[8] - Analogues elliptiques des nombres multizêtas, preprint arXiv:1031.3042, 2013.

[9] A. B. Goncharov, Multiple polylogarithms, cyclotomy and modular complexes, Math. Research Let. 5 (1998), 497-516.

[10] A. B. Goncharov, The dihedral Lie algebras and Galois symmetries of $\pi_{1}^{(\ell)}\left(\mathbb{P}^{1}-\left(\{0, \infty\} \cup m u_{N}\right)\right)$, Duke Math. J. 110 (2001), no. 3, p. 397-487.

[11] R. Hain, letter to P. Deligne, Durham, winter 2009.

[12] R. Hain \& M. Matsumoto, Universal Mixed Elliptic Motives, preprint, February 2012.

[13] Y. Ihara, Some arithmetic aspects of Galois actions in the pro- $p$ fundamental group of $\mathbb{P}^{1}-$ $\{0,1, \infty\}$, in Arithmetic fundamental groups and noncommutative algebra, Proc. Sympos. Pure Math., 70 (2002).

[14] K. Ihara, M. Kaneko \& D. Zagier, Derivation and double shuffle relations for multiple zeta values, Compos. Math. 142 (2006), no. 2, p. 307-338.

[15] F. Martin \& E. Royer, Formes modulaires et périodes, Sém. \& Congrès 12 (2005), SMF, Paris.

[16] J.-G. Luque, J.-C. Novelli \& J.-Y. Thibon, Period polynomials and Ihara brackets, J. Lie Th. 17 (2007), no. 2, p. 229-239.

[17] A. Pollack, Relations between derivations arising from modular forms, senior thesis, 2009.

[18] G. Racinet, Doubles mélanges des polylogarithmes multiples aux racines de l'unité, Publ. Math. de l'IHES no. 95 (2002).

[19] A. Salerno \& L. Schneps, A mould-theoretic proof of the double shuffle Lie algebra structure, preprint, 2014.

[20] L. Schneps, On the Poisson bracket on the free Lie algebra in two generators, J. Lie Th. 16 (2006), no. 1, p. 19-37.

[21] - Double shuffle and Kashiwara-Vergne Lie algebras, J. Algebra 367 (2012), p. 54-74.

[22] - ARI, GARI, Zig and Zag:An introduction to Ecalle's theory of multiple zeta values, preprint arXiv:1507.01534, 2015.

[23] J.-P. Serre, Algèbres de Lie semi-simples complexes, W. A. Benjamin, 1966. 